\section{P-66 SOUTH WEST HOSPICES COLLABORATE ON QUALITY METRICS}

${ }^{1}$ Joan Dee, ${ }^{2}$ John Bailey, ${ }^{3}$ Teresa Coombes. ${ }^{1}$ St Luke's Hospice, Plymouth, UK; ${ }^{2}$ Weston Hospicecare, Weston-super-Mare, UK; ${ }^{3}$ St Margaret's Hospice, Taunton, UK

\subsection{6/bmjspcare-2018-hospiceabs.91}

Background The Directors of Care of eight south-west hospices identified a lack of consensus over measures that identify a hospice quality service. The Quality Leads of these services were asked to develop potential measures of quality and submit recommendations.

Aim To develop a set of metrics that reflects and measures quality identifying any potential learning (including in the narrative behind the figures) thus improving care provision.

Method A Quality Leads Forum was set up and developed a set of metrics that reflect and measure quality and identifies areas of learning: including topics such as falls, drug errors, themes from feedback and complaints.

Results We agreed to use the National Quality Board definition of quality as the framework for developing our metrics. It became clear that although hospices often measure similar things we do not always do so in the same way. In order to address the differences it is necessary to develop universal standards for each measure identified. Over the next 12 months we will develop standard measurements that will include: healed pressure injuries, the implementation of OACC, response to referrals and delayed discharges. Following this we plan to set up a process of peer review utilising these metrics. Conclusions Although there are many differences in the services hospices provide and the standards they set, the development of this metrics so far would indicate that there are ways that we can work collaboratively in order to evidence the quality of the care we provide. A further unintentional benefit of the group/metrics is the ability to benchmark with peers and share learning/opportunities for service improvement projects as spin offs. We identified common challenges (e.g. transport) and now have a platform giving clear guidance on where to concentrate our improvement plans.

\section{P-67 USING THE INTEGRATED PALLIATIVE OUTCOME SCORE DATA TO TRANSFORM CLINICAL CARE}

Luke Feathers, Gemma Miller, Stacey Hubber. LOROS Hospice, Leicester, UK

\subsection{6/bmjspcare-2018-hospiceabs.92}

Background Since 2015 LOROS has been using the Integrated Palliative Outcome Score (IPOS) in our clinical services. We present how we use IPOS data in our MDT meetings and feed back to clinical departments on a regular basis through dashboards.

In partnership with a small IT company (LHITS) and St Barnabas Hospice, Lincolnshire, we developed an IPOS reporting tool to extract data from SystmOne.

Aims Enabling IPOS data to be used across departments to make informed decisions about patient care in MDT meetings. Providing feedback to departments on outcome measures to show their impact on care. Demonstrating to stakeholders the difference that the hospice has made for patients.

Methods Collaboratively we devised a data extraction tool that from SystmOne data produces a patient level dashboard for MDTs, showing changes in IPOS scores across time, and a service level dashboard showing scores at change of phase.
Results Being able to see IPOS data graphically (radar, bar and heat-map charts) has enabled clinical teams to see where to focus treatment, whether around physical, psychological, spiritual or practical areas. Clinical department reports are used to identify areas where care is already improving patient scores, and also to concentrate on areas needing a different approach to support patients.

Conclusion We are addressing the practical issue of how best to use IPOS data both clinically and managerially, aiming to transfer data from the electronic record with a 'single button' methodology. This allows patient reported data to both improve clinical decision making in a team setting and department managers to focus on areas of difficulty for their patient cohorts. Sharing our experience with other hospice providers will enable this approach to be used more widely for the benefit of patients and services.

\section{P-68 $\quad$.. TO PUBLISH OR BE DAMNED: DECISION-MAKING AROUND PATIENT CONFIDENTIALITY IN CASE STUDY RESEARCH}

Steve Nolan. Princess Alice Hospice, Esher, Surrey

\subsection{6/bmjspcare-2018-hospiceabs.93}

Background As a form of qualitative research, case studies offer practitioners the possibility of contributing from their own clinical experience to innovating palliative care practice (Campbell, 2013). However, clinical information must be used sensitively and must protect patient confidentiality (UK Board of Healthcare Chaplaincy, 2014). Best practice dictates gaining informed consent; however, where consent is either not possible or would not be appropriate, the anonymisation of identifying details is recognized as an alternative way to protect confidentiality. But practitioner-researchers remain ethically responsible for protecting confidentiality.

Aim To produce an algorithm that can guide would-be case study practitioner-researchers through the decision-making process about seeking informed consent and how (and whether) to proceed without it.

Methods The issue was first discussed by the Hospice Ethics Committee. This identified the range of concerns the algorithm needed to address. These concerns were discussed further by the Hospice Research Committee, which requested a draft algorithm. The draft was presented and refinements were recommended. Once accepted, the algorithm was tested against a request by the Hospice Chaplain to publish a case study.

Results An algorithm was produced that guides the would-be practitioner-researcher through the decision-making process about seeking informed consent towards case study publication. Beginning with the question, 'Is the subject able to give consent?' the flowchart guides practitioner-researchers step-bystep through a series of 'pinch-points' in making their decision, highlighting key questions that need to be considered. These include: whether the subject has capacity; the appropriateness of requesting consent from the subject or their relative; whether the case can be suitability anonymised; and how to respond to organisational peer-review.

Conclusions The algorithm is an effective tool that guides practitioner-researchers step-by-step through the process of deciding whether they can proceed to publish clinical case material while properly respecting patients' confidentiality. 